\title{
Nano-impacts of Bifunctional Organic Nanoparticles
}

\author{
X-F Zhou, W Cheng and R G. Compton*
}

*Corresponding author

Department of Chemistry, Physical and Theoretical Chemistry Laboratory, Oxford University, South Parks Road, Oxford, OX1 3QZ, United Kingdom.

Fax: +44 (0) 1865 275410; Tel: +44 (0) 1865275957.

Email: richard.compton@chem.ox.ac.uk 


\begin{abstract}
The synthesis and characterization of Oil Blue Dye nanoparticles is reported along with their use for nano-impacts experiments in aqueous solution. The latter reveal current spikes corresponding to quantitative two electron reductions due to the reduction of the quinone groups and quantitative two electron oxidations from the 1, 4-phenylenediamine groups presented in the Oil Blue Dye molecules within the nanoparticles. In both cases, the oxidation or reduction leads to size distributions in good agreement with independent measurements made using dynamic light scattering showing that the redox events accompanying the nano-impacts lead to the full dissolution of the nanoparticles.
\end{abstract}

\title{
Keywords
}

Nano-impacts; Nanoparticles; Oil Blue Dye; Cyclic voltammetry; Chronoamperometry 


\section{Introduction}

We have recently shown that so-called 'nano-impacts' can be used for the identification and sizing of nanoparticles suspended in aqueous solution ${ }^{1-8}$. In these experiments nanoparticles move randomly in solution by virtue of their Brownian motion and from time to time come into contact with a microelectrode located within the solution. These encounters may lead to adsorption or alternatively the nanoparticles may simply rebound from the electrode surface. Alternatively, if the electrode is held at a suitable potential the nanoparticles can become quantitatively oxidized ${ }^{9}$. For example, in the case of silver nanoparticles (NPs)

$$
\operatorname{Ag}_{\mathrm{n}}(\mathrm{NP})-\mathrm{ne}^{-} \rightarrow \mathrm{nAg}^{+}(\mathrm{aq})
$$

In this case, the 'nano-impacts' of individual nanoparticles can be observed as 'spikes' in a current time transient. The charge passed in each spike goes an immediate indication of the number of atoms in the nanoparticles being oxidized by virtue of Faraday 1st law:

$$
\mathrm{Q}=\text { ne }
$$

where $\mathrm{Q}$ is the integrated charge, e is the electric charge $\left(1.6 \times 10^{-19} \mathrm{C}\right)$ and $\mathrm{n}$ is the number of atoms in the nanoparticles. In this manner, metal nanoparticles such as $\mathrm{Ag}^{1}, \mathrm{Ni}^{2}, \mathrm{Au}{ }^{3}$ and $\mathrm{Cu}^{5}$ have been sized and the particle size distribution shown to be in excellent agreement with independent measurements using in-situ dynamic light scattering and nanoparticles tracking analysis or ex-situ electron microscopy.

Work on 'nano-impacts' has previously focused to date primarily on metal nanoparticles ${ }^{1-7}$, where it has been shown that in addition to size distributions ${ }^{1}$, concentrations ${ }^{2}$ and information about the state of agglomeration ${ }^{4}$ or aggregation ${ }^{6}$ of the nanoparticles can be quantitatively measured. Work has also been done on the metal oxide $\mathrm{Fe}_{3} \mathrm{O}_{4}$ $\left(\mathrm{Fe}(\mathrm{II})_{2} \mathrm{Fe}(\mathrm{III}) \mathrm{O}_{4}\right){ }^{10}$ where both reductive and oxidative transients are seen. The former 
correspond to the reduction of $\mathrm{Fe}(\mathrm{III})$ to $\mathrm{Fe}$ (II) and the latter to the oxidation of $\mathrm{Fe}$ ( II ) to $\mathrm{Fe}(\mathrm{III})$. For both types of transient the quantitative dissolution of the $\mathrm{Fe}_{3} \mathrm{O}_{4}$ nanoparticles was inferred.

Hitherto reports of nano-impacts experiments using organic nanoparticles are restricted to just one example, that of the reduction of indigo nanoparticles ${ }^{8,11}$. In the current paper we present a second example namely that of nanoparticles of Oil Blue Dye (OBD). In addition to sizing these nanoparticles electrochemically using nano-impacts we take advantage of their bifunctional character. In particular they have both quinone and phenylenediamine groups, such that the nanoparticles can undergo both oxidation and reduction in nano-impacts experiments as shown in Scheme 1.

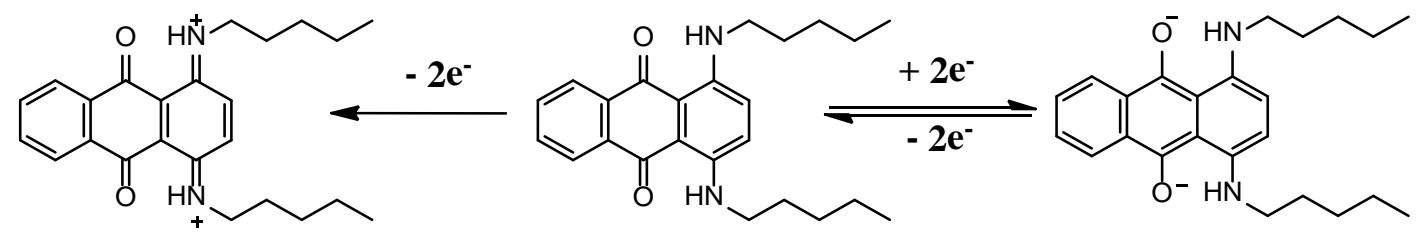

Scheme 1. Likely mechanism of Oil Blue Dye reduction and oxidation

By comparison of the charge passed together with DLS analysis in both oxidation and reduction further strong evidence is deduced for the quantitative electroanalysis of organic nanoparticles during nano-impacts. 


\section{Experimental}

\subsection{Chemicals and Materials}

Oil Blue N (dye content 96\%), acetone (ACS reagent, $\geq 99.5 \%$ ), acetonitrile (anhydrous, 99.8\%), tetra-n-butylammoniumperchlorate (TBAP, electrochemical grade, 99\%) were purchased from Aldrich and used as received. The phosphate buffer solution $(\mathrm{pH}=6.8)$ was composed of $50 \mathrm{mM}$ monobasic and dibasic potassium phosphates, $0.1 \mathrm{M} \mathrm{KCl}$ used as further supporting electrolyte. Unless stated otherwise, all chemicals in this work were acquired from Sigma-Aldrich. All solutions were made up using ultrapure water of resistivity $18.2 \mathrm{M} \Omega \mathrm{cm}$ (Millipore) at $25^{\circ} \mathrm{C}$ and degassed thoroughly with $\mathrm{N}_{2}$ (oxygen-free, BOC Gases plc) before use.

\subsection{Synthesis and Characterization of OBD Nanoparticles}

OBD (Oil Blue N Dye) nanoparticles were synthesised by the re-precipitation method ${ }^{12}$. OBD was dissolved in $20 \mathrm{~mL}$ acetone, giving an OBD concentration of $2.5 \mathrm{mM}$. Then $1 \mathrm{~mL}$ OBD/acetone solution was injected into deionized water and thoroughly mixed by magnetic vortexing stirring at $800 \mathrm{rpm}$. The resulting mixture was a dark blue, transparent dispersion. Then, it was subject to sonication for 20 minutes at room temperature. The size distributions of synthesised OBD nanoparticles were measured by dynamic light scattering (Malvern Instruments Ltd, UK).

\subsection{Electrochemical Characterization of OBD and OBD Nanoparticles}

The electrochemical experiments were conducted at room temperature with a double Faraday cage with a three electrode system using an Autolab II potentiostat (Metrohm-Autolab BV, Netherlands). For cyclic voltammetry in organic solvents and drop casting experiments in 
aqueous solution, a glassy carbon (GC, diameter $=3.0 \mathrm{~mm}$ ) electrode was used as a working electrode, a saturated calomel electrode (SCE) as a reference (Radiometer, Copenhagen), and a platinum mesh as a counter electrode.

\subsection{Nano-impacts of OBD Nanoparticles}

For electrochemical sizing and chronoamperometry, a carbon microelectrode (diameter $=11$ $\mu \mathrm{m})$ was used as working electrode with the same reference and counter electrodes as above. The micro carbon electrode diameter was calibrated electrochemically by analysing the steady state voltammetry of a $2.0 \mathrm{mM}$ ferrocene in acetonitrile containing $0.1 \mathrm{M}$ TBAP, using a diffusion coefficient for ferrocene of $2.30 \times 10^{-9} \mathrm{~m}^{2} \mathrm{~s}^{-1}$ at $298 \mathrm{~K}^{13}$. The program "Signal Counter” (developed by Dr. D Omanovic' at Center for Marine and Environmental Research Zagreb, Croatia) was used for impact spikes identification and individual spike area determination. OriginPro 8.5.1 was applied for electrochemical data analysis and nanoparticle sizing. 


\section{Result and Discussion}

\subsection{Electrochemical Characterization of Molecular OBD Dissolved in Acetonitrile}

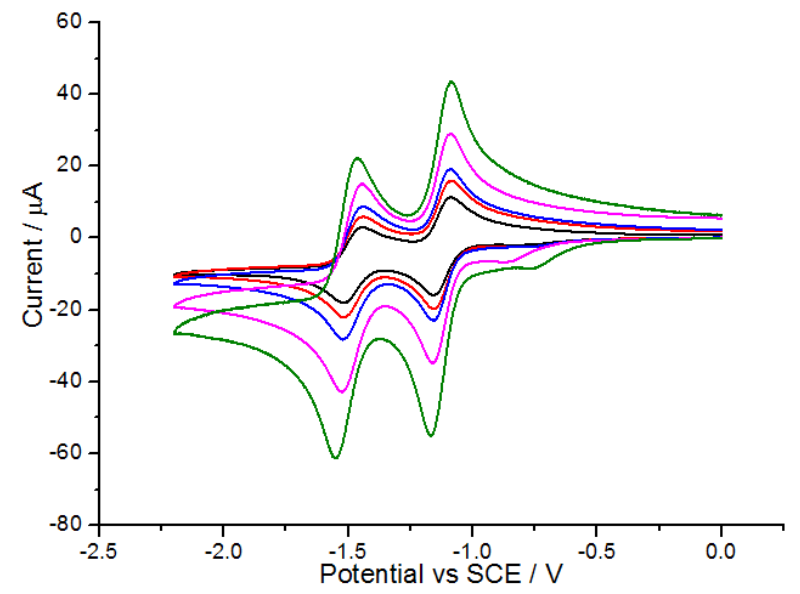

Figure 1.The cyclic voltammetric reductive response of $1.0 \mathrm{mM}$ OBD in acetonitrile with $0.10 \mathrm{M}$ TBAP as supporting electrolyte at scan rates of $50 \mathrm{mV} / \mathrm{s}$ (black), $75 \mathrm{mV} / \mathrm{s}$ (red), $100 \mathrm{mV} / \mathrm{s}$ (blue), $200 \mathrm{mV} / \mathrm{s}$ (magenta) and $500 \mathrm{mV} / \mathrm{s}$ (green)

A bare glassy carbon electrode was immersed in $1.0 \mathrm{mM}$ OBD in acetonitrile solution with 0.10 M TBAP as supporting electrolyte. The solution was degassed to remove electroactive dissolved oxygen. Cyclic voltammetry was recorded at different scan rates from $50 \mathrm{mV} / \mathrm{s}$ to $500 \mathrm{mV} / \mathrm{s}$ as shown in Figure 1. The cyclic voltammetry shows two separate cathodic peaks, likely responding to formation of mono and dianions (Scheme 2) respectively through two single-electron transfer processes. This is a similar observation that seems for the reduction of molecular anthraquinone ${ }^{14-16}$. At slow scan rates, peak separations of the first pair of redox peaks are ca. $60 \mathrm{mV}$, indicating an electrochemically reversible process for the first electron transfer of OBD. 


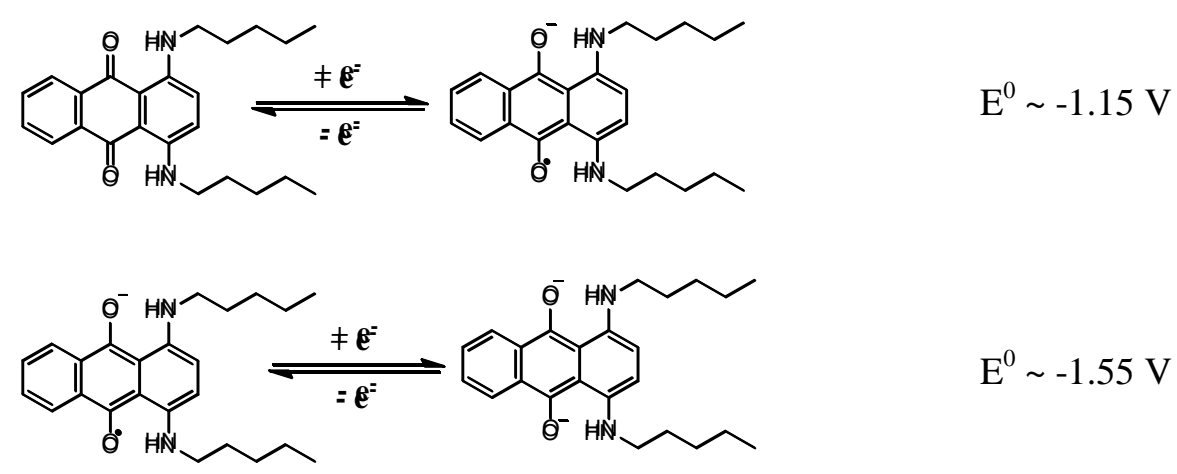

Scheme 2. Likely mechanism of OBD reduction

To analyze the data in Figure 1 to give the diffusion coefficient of OBD in acetonitrile, we assumed that the first electron reduction of $\mathrm{OBD}$ is fully reversible and plotted the peak current versus square root of scan rate as shown in Figure 2 (measured for the $-1.15 \mathrm{~V}$

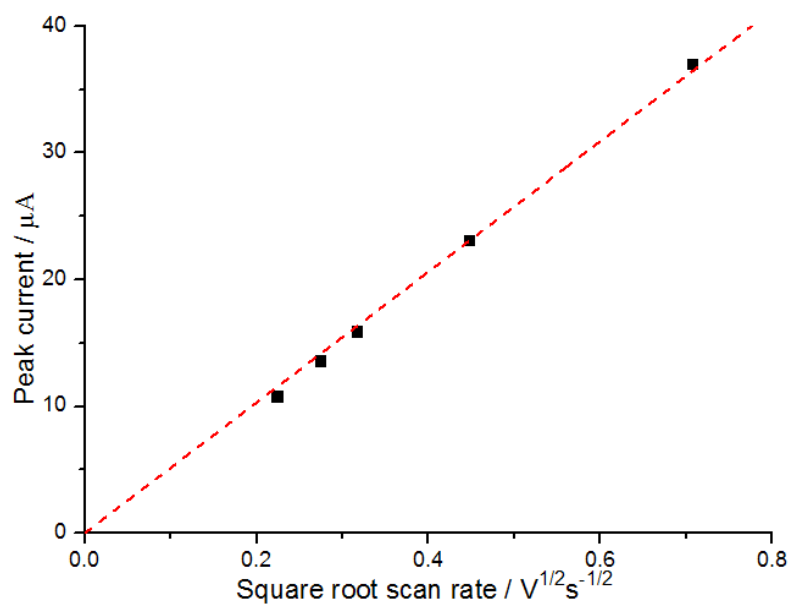

Figure 2. First reduction peak current verses square root of scan rate from $50 \mathrm{mV} / \mathrm{s}$ to $500 \mathrm{mV} / \mathrm{s}$ peak). By using the Randles-Sevcik equation, the diffusion coefficient $\mathrm{D}$ of OBD was found to be $7.3 \times 10^{-6} \mathrm{~cm}^{2} \mathrm{~s}^{-1}$.

$$
I_{P}=2.69 \times 10^{5} A D^{1 / 2}[A]_{b u l k} v^{1 / 2}
$$

where $I_{P}$ is the peak current, $A$ is the surface area of the glassy carbon macroelectrode, $[A]_{\text {bulk }}$ is the concentration of $\mathrm{OBD}$ in acetonitrile and $v$ is the scan rate.

Next, the oxidative cyclic voltammetry of OBD was conducted (Figure 3). In order to explore the mechanism of OBD oxidation reaction, the vertex potentials for cyclic voltammetry were set as $+1.4 \mathrm{~V}$ or $+0.95 \mathrm{~V}$. 
Cyclic voltammetry set at $+0.95 \mathrm{~V}$ as the vertex potential at different scan rates from $50 \mathrm{mV} / \mathrm{s}$ up to $500 \mathrm{mV} / \mathrm{s}$ is shown in Figure 3(a). Peak separation between forward and backward peaks is around $62 \mathrm{mV}$, showing a likely electrochemically reversible oxidation of OBD to OBD monovalent cations corresponding a single electron transfer process. Next, the cyclic voltammetry with $+1.40 \mathrm{~V}$ as the vertex potential shows a pair of separate redox peaks as shown in Figure 3(b). The positions of the forward and backward peaks for the second oxidation shift with scan rates, indicating that the oxidation of OBD monovalent cations to OBD bivalent cations matches along with a single electron transfer process. However, the position of the backward peaks does not show a reversible type of redox shape, indicating that there might be other products generated during the reduction of OBD bivalent cations. The likely mechanism is shown in Scheme 3 and is that expected for phenylenediamines ${ }^{17}$.
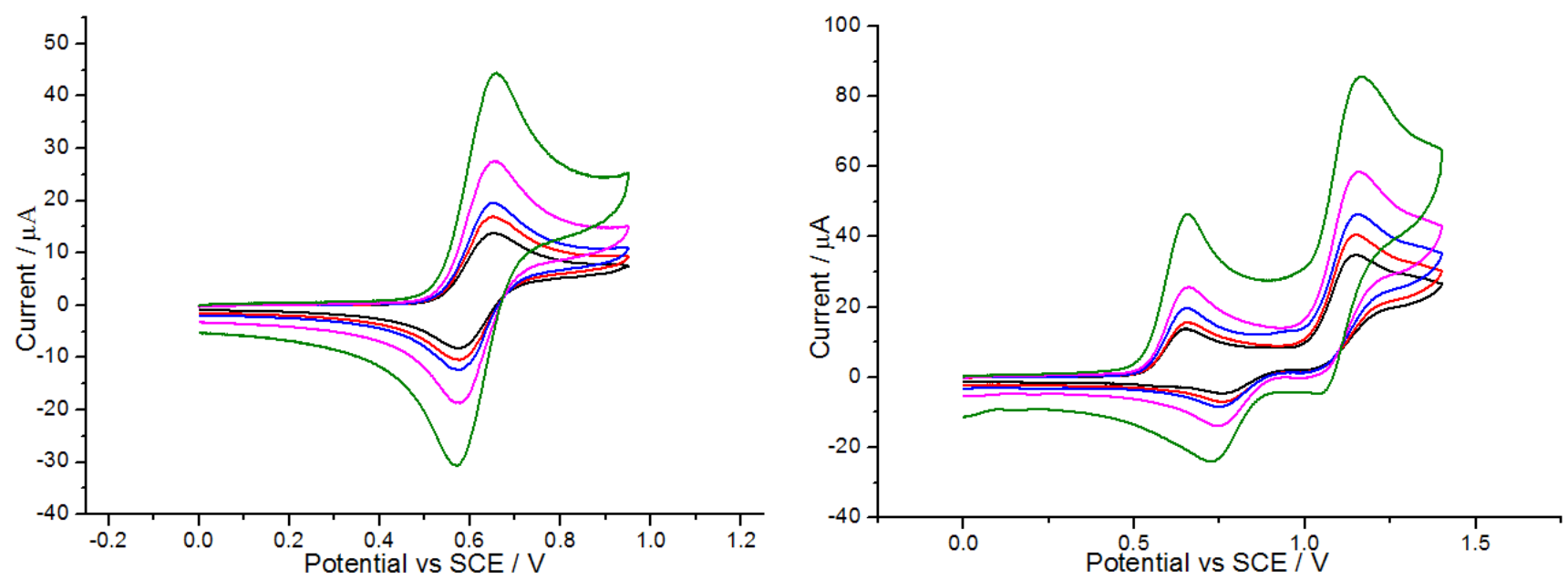

Figure 3.The cyclic voltammetric oxidative response of $1.0 \mathrm{mM}$ OBD oxidation in acetonitrile with $0.10 \mathrm{M}$ TBAP as supporting electrolyte at scan rate $50 \mathrm{mV} / \mathrm{s}$ (black), $75 \mathrm{mV} / \mathrm{s}$ (red), $100 \mathrm{mV} / \mathrm{s}$ (blue), $200 \mathrm{mV} / \mathrm{s}$ (magenta) and $500 \mathrm{mV} / \mathrm{s}$ (green) with vertex potential of cyclic voltammetry set as $+1.4 \mathrm{~V}$ (a) and $+0.95 \mathrm{~V}$ (b)

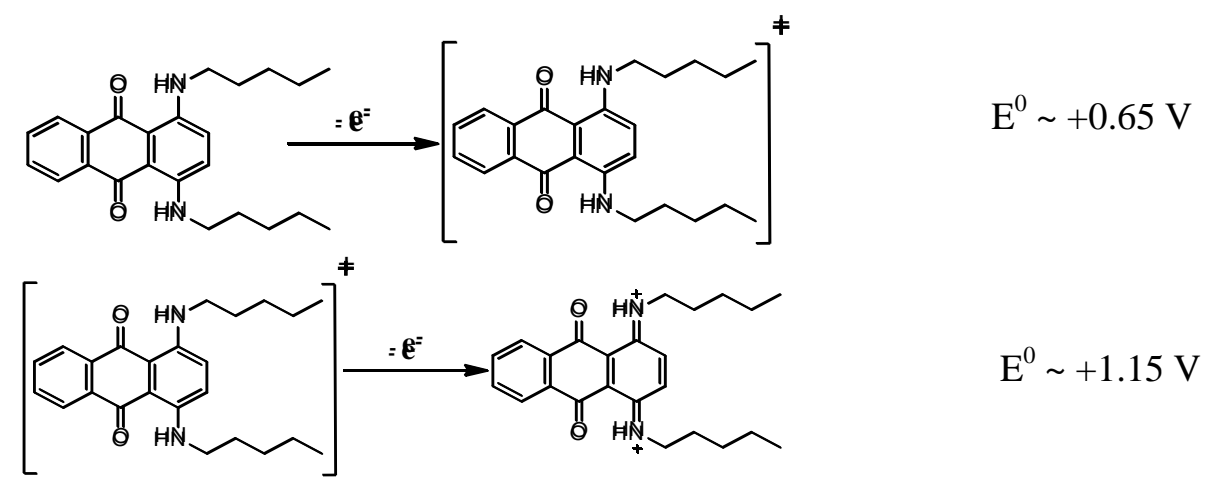

Scheme 3. Likely mechanism of OBD oxidation 


\subsection{Electrochemical Characterization of OBD Nanoparticles in Aqueous Solution}

To estimate the reduction and oxidation potentials of OBD NPs for subsequent nano-impacts experiments, a glassy carbon macroelectrode was modified by dropping a known amount of a OBD NPs suspension $\left(2 \times 10^{-4} \mathrm{~mol} / \mathrm{L}\right)$ onto the surface and letting the electrode dry under nitrogen. Cyclic voltammetry was then recorded with the modified electrode immersed in PBS buffer. A significant reduction peak was observed at $-0.75 \mathrm{~V}$, which can be attributed to the reduction of the quinone group via a two electron transfer process ${ }^{14,18-20}$, and an oxidation peak at $+0.95 \mathrm{~V}$ indicating the oxidation of OBD NPs electrochemical response as shown in Figure 4. This peak is similar in size to the reduction peak observed.
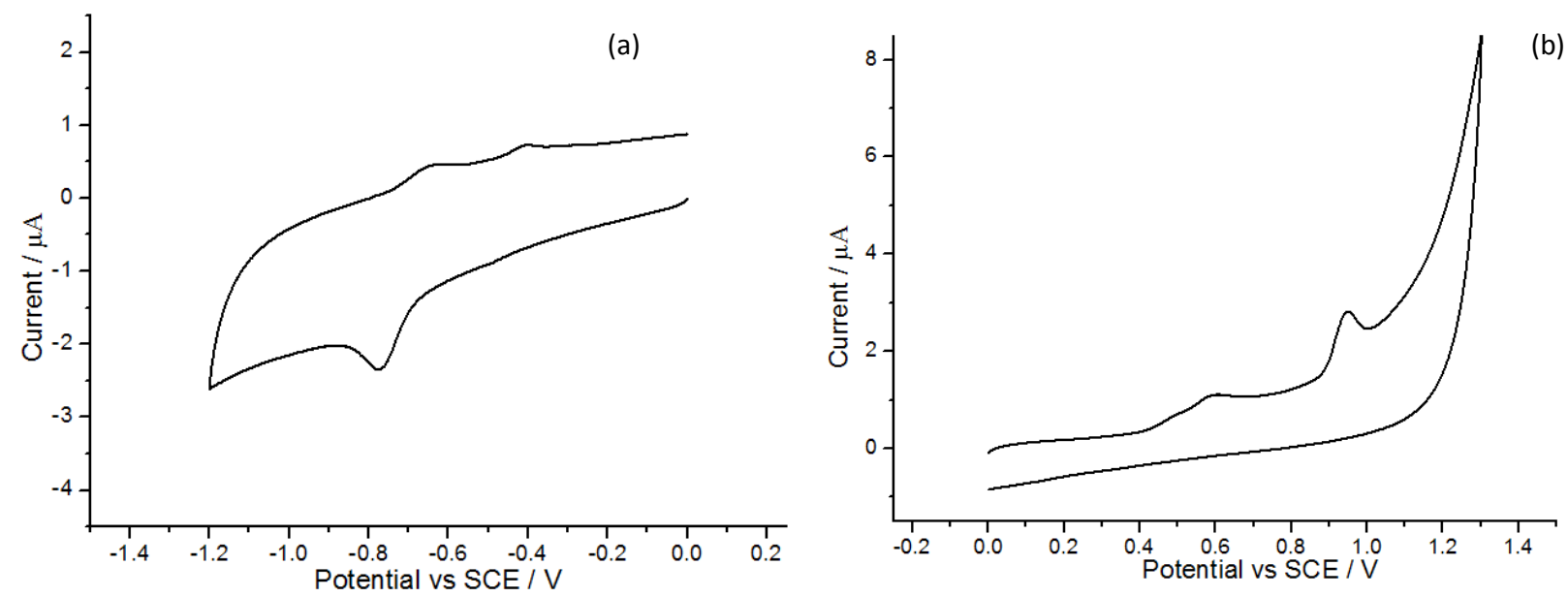

Figure 4 Cyclic voltammetric response of an OBD NPs modified GC electrode in a $0.1 \mathrm{M}$ PBS buffer $(\mathrm{pH}=6.8)$ at a scan rate of $50 \mathrm{mV} / \mathrm{s}$ (a) Reduction and (b) Oxidation

A smaller oxidation peak at $+0.60 \mathrm{~V}$ was also observed, which likely corresponds to the partial one electron oxidation of OBD nanoparticles on the outer surface of the electrode ${ }^{21}$, giving that the oxidation may be size dependent ${ }^{22}$. To ensure complete oxidation of the OBD 
nanoparticles thus allowing their accurate sizing, the potential used for oxidation of OBD NPs in nano-impact experiments reported below was set at $+1.0 \mathrm{~V}$.

\subsection{Nano-impacts of OBD Nanoparticles}

A carbon microelectrode was placed in 0.1 M PBS buffer solution $(\mathrm{pH}=6.8)$ and a known concentration of pre-dispersed OBD NPs added. Under potentiostatted conditions at $-0.80 \mathrm{~V}$ vs. SCE, reductive (Faradaic) spikes from individual OBD NPs were observed (Figure 5a), showing that direct reduction of individual OBD nanoparticles during random collision events can be observed and quantified. In these studies, 52 chronoamperograms of $5 \mathrm{~s}$ duration were recorded at a potential of $-0.80 \mathrm{~V}$, yielding 253 spikes for OBD NPs reduction reaction and the potential of $+1.0 \mathrm{~V}$ was applied over 5 s for 58 times, yielding 98 spikes in total for OBD NPs oxidation reaction.
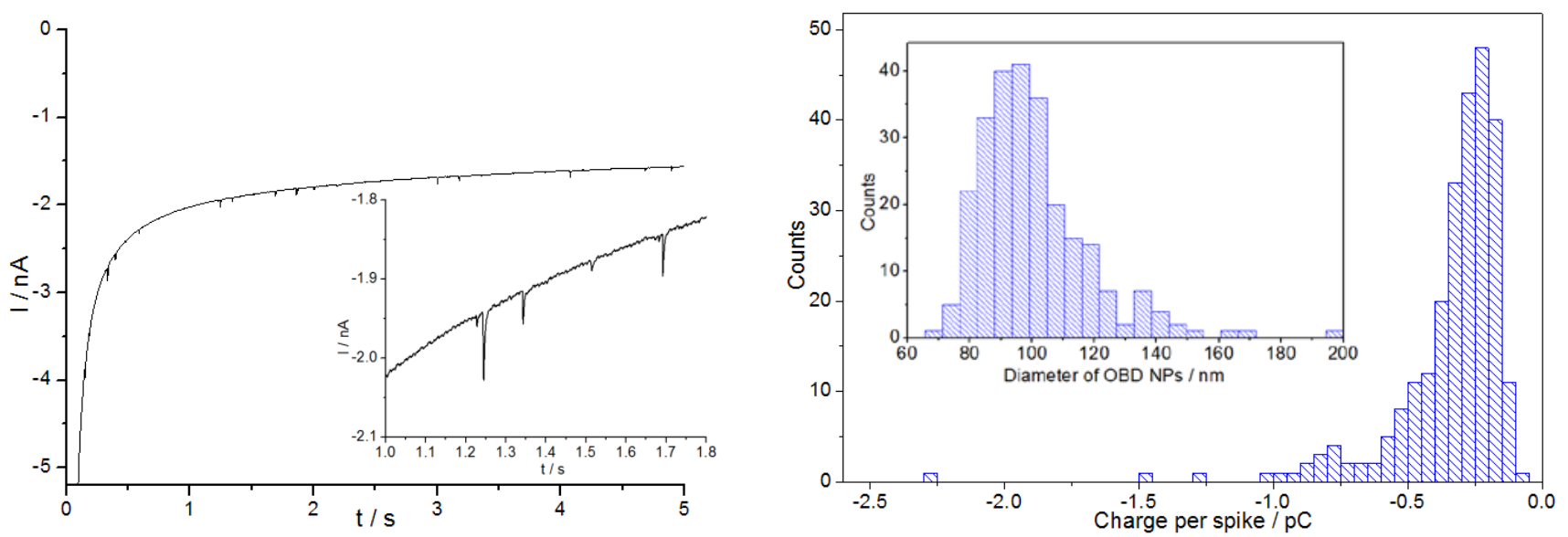

Figure 5 a) Chronoamperometric profiles showing reductive Faradaic spikes of OBD NPs in PBS buffer $(\mathrm{pH}=6.8)$ at $-0.80 \mathrm{~V}$ vs SCE. The inset showing the detailed impact spikes. b) Histogram showing the OBD NPs reductive charge and the inset showing size distribution.

Reductive spikes of OBD NPs at $-0.80 \mathrm{~V}$ are shown in Figure $5 \mathrm{a}$, showing the reduction of individual OBD NPs during random collisions with the surface of the carbon microelectrode. The onset of these Faradaic spikes was found to be dependent on reduction potential. Only a very few spikes were observed by reducing reduction potential such as $-0.30 \mathrm{~V}$, confirming 
that the spikes at $-0.80 \mathrm{~V}$ result from the Faradaic reduction of OBD NPs. The charge passed during reduction of individual OBD NPs was plotted by integrating the individual spikes as shown in Figure 5b. Then, we quantified the size of OBD NPs by using the equation between diameter and charge of single OBD NPs. Assuming OBD NPs are spherical, the charge Q passed as a result of complete reduction of single OBD NPs is given by:

$$
D_{n p}=2 \times \sqrt[3]{\frac{3 M Q}{4 n F \pi \rho}}
$$

where $\mathrm{D}_{\mathrm{np}}$ is the diameter of OBD NPs, M is the molar mass of OBD, $\rho$ is the density of OBD and $\mathrm{F}$ is the Faraday constant. The parameter $\mathrm{n}$ is the number of electrons transferred of OBD NPs reduction $(n=2)$ as discussed above. The size distribution derived by analysis of the 253 reduction spikes is shown in Figure 5b (inset), giving the mean diameter and standard deviation of OBD NPs as $100 \pm 18 \mathrm{~nm}$.
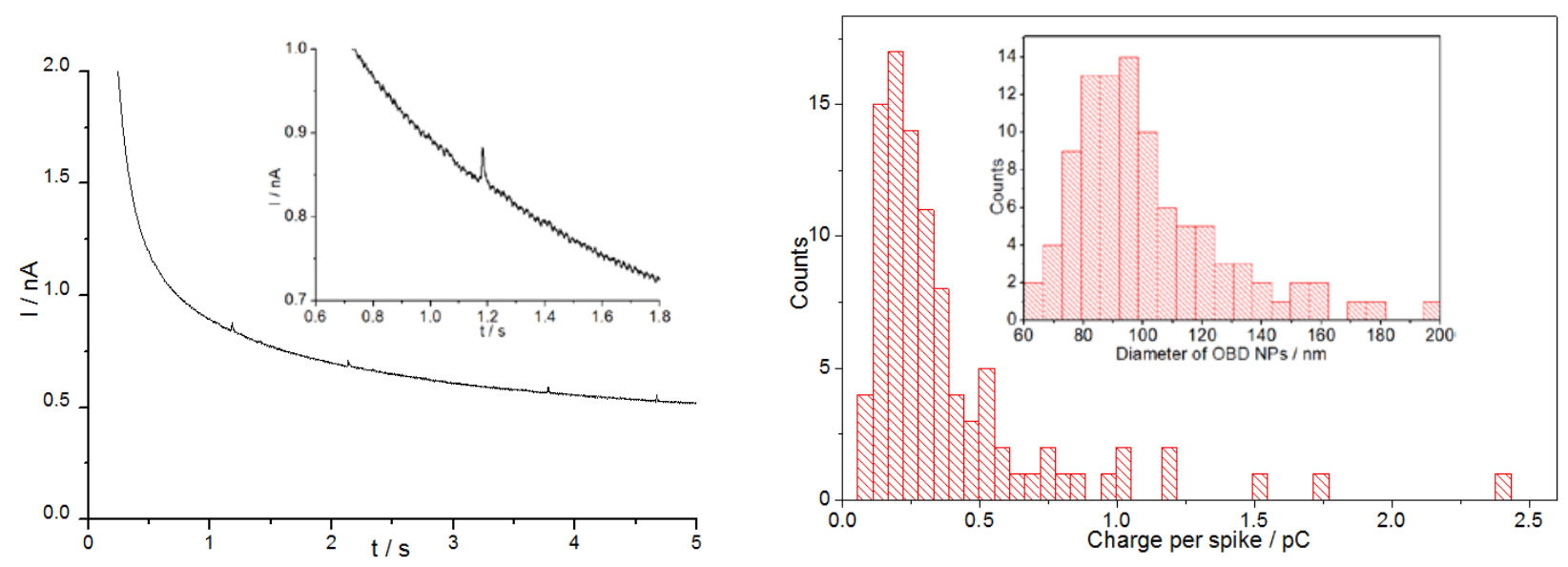

Figure 6 a) Chronoamperometric profiles showing oxdative Faradaic spikes of OBD NPs in PBS buffer ( $\mathrm{pH}=6.8$ ) at $+1.00 \mathrm{~V}$ vs SCE. The inset showing the detailed impact spikes. b) Histogram showing the OBD NPs oxidative charge and the inset showing size distribution.

Similarly, under potentiostatted conditions at $+1.00 \mathrm{~V}$ vs. SCE, oxidative (Faradaic) spikes from individual OBD NPs were also observed (Figure 6a), showing for the first time that 
direct oxidation of individual organic nanoparticles is feasible. The size distribution of OBD NPs can also be determined by analysing the Faradaic charge from the oxidative spikes of individual OBD NPs, as discussed above. The 98 impact spikes observed in anodic chronoamperograms were analysed using the same equation as above. The size distribution shows the mean diameter and standard deviation as $102 \pm 26 \mathrm{~nm}$ (Figure 6b), which has an excellent agreement with the sizing from nano-impacting reduction.

The oxidation spikes (Figure 6a) show similar shape to the reduction spikes (Figure 5a). However, under the same concentration of OBD NPs, the frequency of reductive spikes is higher than that of the oxidative spikes, which may be due to the electrode surface being blocked by oxidative products adsorptively, physically or covalently ${ }^{23}$, while in contrast there is clean reductive dissolution of the OBD nanoparticles.

\subsection{Electrochemical Sizing and DLS Sizing of OBD Nanoparticles}

To further verify our results from electrochemical sizing through the reduction and oxidation, we used dynamic light scattering (DLS) to characterize the same batch of OBD NPs. Both reduction and oxidation of OBD NPs yield the same size distributions which have an excellent agreement with the results obtained by DLS analysis, as shown in Figure 7. Thus we show for the first time that the size of biofunctional organic nanoparticles can be quantitively determined by both direct oxidation and reduction by the nano-impact method. 


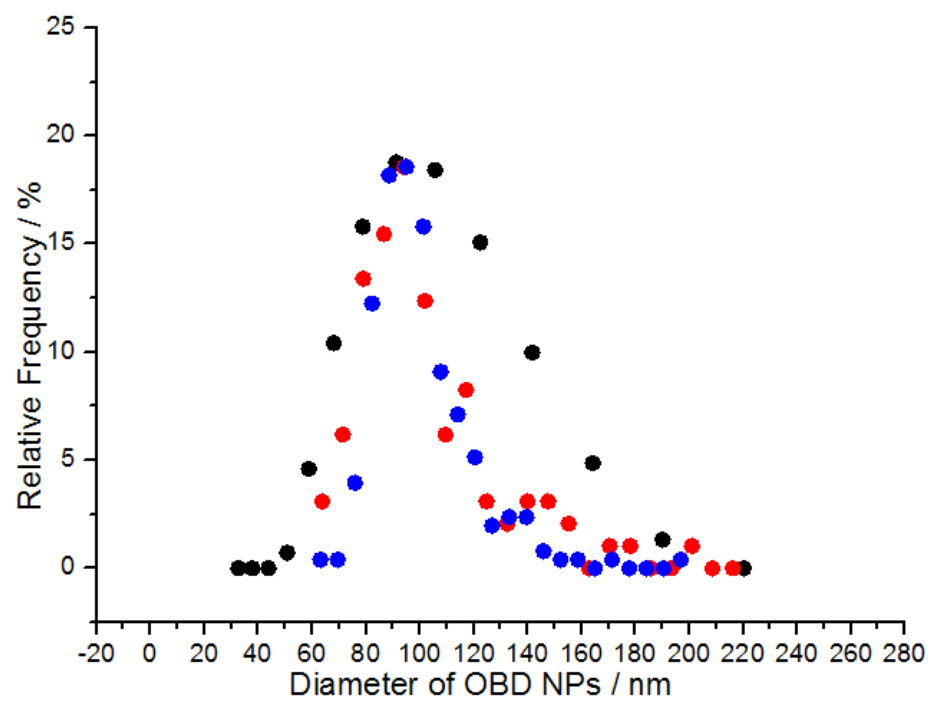

Figure 7.Size distribution of OBD NPs obtained from electrochemical sizing of nano-impacting experiments (blue dots: reduction; red dots: oxidation) and DLS analysis (black dots).

\section{Conclusions}

Herein, we use electrochemical methods to quantify the size of bifunctional organic nanoparticles for the first time by both direct reduction and oxidation routes of individual bifunctional organic nanoparticles. The agreement between the sizing distributions carried out via oxidation and reduction provides further evidence for the quantitative electroanalysis of nanoparticles during nano-impacts.

\section{Acknowledgements}

The research leading to these results has received funding from the European Research Council under the European Union’s Seventh Framework Programme (FP/2007-2013) / ERC Grant Agreement n. [320403]. We also thank Dr. D. Omanovic' (Center for Marine and Environmental Research Zagreb, Croatia) for developing Signal Counter software for data analysis and the Leverhulme Trust for support for W.C 


\section{References}

1. Y. G. Zhou, N. V. Rees and R. G. Compton, Angew Chem Int Ed, 2011, 50, 4219-4221.

2. E. J. E. Stuart, Y. G. Zhou, N. V. Rees and R. G. Compton, Rsc Adv, 2012, 2, 6879-6884.

3. Y. G. Zhou, N. V. Rees, J. Pillay, R. Tshikhudo, S. Vilakazi and R. G. Compton, Chem Commun, 2012, 48, 224-226.

4. J. Ellison, K. Tschulik, E. J. E. Stuart, K. Jurkschat, D. Omanovic, M. Uhlemann, A. Crossley and R. G. Compton, ChemistryOpen, 2013, 2, 69-75.

5. B. Haddou, N. V. Rees and R. G. Compton, Phys Chem Chem Phys, 2012, 14, 13612-13617.

6. E. J. E. Stuart, N. V. Rees, J. T. Cullen and R. G. Compton, Nanoscale, 2013, 5, 174-177.

7. E. J. E. Stuart, K. Tschulik, D. Omanovic, J. T. Cullen, K. Jurkschat, A. Crossley and R. G. Compton, Nanotechnology, 2013, 24.

8. W. Cheng, X. F. Zhou and R. G. Compton, Angew Chem Int Ed, 2013, 52, 12980-12982.

9. N. V. Rees, Y. G. Zhou and R. G. Compton, Rsc Adv, 2012, 2, 379-384.

10. K. Tschulik, B. Haddou, D. Omanovic, N. V. Rees and R. G. Compton, Nano Res, 2013, 6, 836841.

11. W. Cheng, C. Batchelor-McAuley and R. G. Compton, ChemElectroChem, 2014. doi: 10.1002/celc. 201300233

12. J. Suk and A. J. Bard, J Solid State Electr, 2011, 15, 2279-2291.

13. E. I. Rogers, D. S. Silvester, D. L. Poole, L. Aldous, C. Hardacre and R. G. Compton, J Phys Chem C, 2008, 112, 2729-2735.

14. C. Batchelor-McAuley, B. R. Kozub, D. Menshykau and R. G. Compton, J Phys Chem C, 2011, 115, 714-718.

15. J. Katsumi, T. Nakayama, Y. Esaka and B. Uno, Anal Sci, 2012, 28, 257-265.

16. I. Piljac and R. W. Murray, J Electrochem Soc, 1971, 118, 1758.

17. X. B. Ji and R. G. Compton, Anal Sci, 2007, 23, 1317-1320.

18. M. A. G. Trindade and M. V. B. Zanoni, Sensor Actuat B-Chem, 2009, 138, 257-263.

19. B. Zinger, J Electroanal Chem, 1988, 239, 209-225.

20. M. A. G. Trindade and M. V. B. Zanoni, Electroanalysis, 2007, 19, 1901-1907.

21. A. Adenier, M. M. Chehimi, I. Gallardo, J. Pinson and N. Vila, Langmuir, 2004, 20, 8243-8253.

22. O. S. Ivanova and F. P. Zamborini, J Am Chem Soc, 2010, 132, 70.

23. E. O. Barnes and R. G. Compton, J Electroanal Chem, 2013, 693, 73-78. 